Network Working Group

Request for Comments: 4559

Category: Informational
K. Jaganathan

L. Zhu

J. Brezak

Microsoft Corporation June 2006

\title{
SPNEGO-based Kerberos and NTLM HTTP Authentication \\ in Microsoft Windows
}

\section{Status of This Memo}

This memo provides information for the Internet community. It does not specify an Internet standard of any kind. Distribution of this memo is unlimited.

Copyright Notice

Copyright (C) The Internet Society (2006).

Abstract

This document describes how the Microsoft Internet Explorer (MSIE) and Internet Information Services (IIS) incorporated in Microsoft Windows 2000 use Kerberos for security enhancements of web transactions. The Hypertext Transport Protocol (HTTP) auth-scheme of "negotiate" is defined here; when the negotiation results in the selection of Kerberos, the security services of authentication and, optionally, impersonation (the IIS server assumes the windows identity of the principal that has been authenticated) are performed. This document explains how HTTP authentication utilizes the Simple and Protected GSS-API Negotiation mechanism. Details of Simple And Protected Negotiate (SPNEGO) implementation are not provided in this document.

Table of Contents

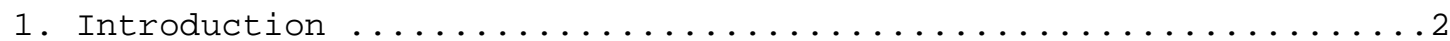

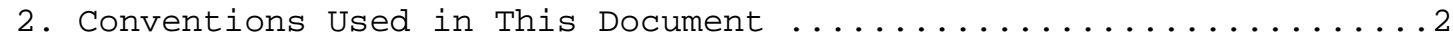

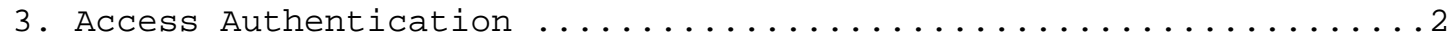

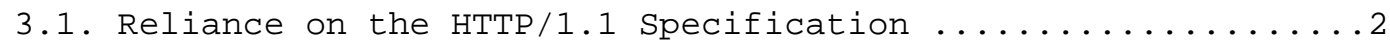

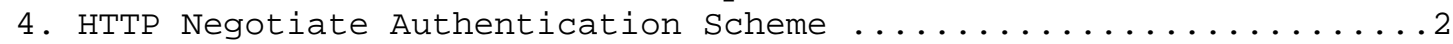

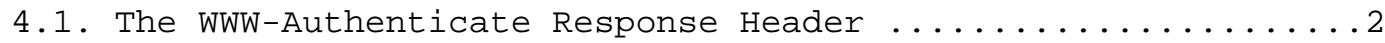

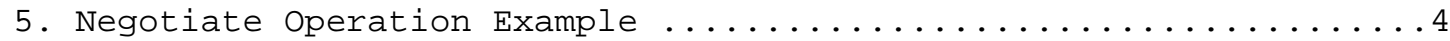

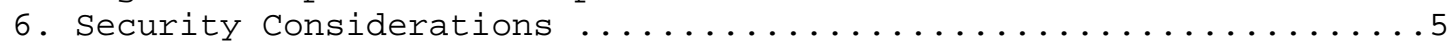

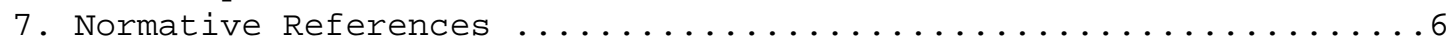




\section{Introduction}

Microsoft has provided support for Kerberos authentication in Microsoft Internet Explorer (MSIE) and Internet Information Services (IIS), in addition to other mechanisms. This provides the benefits of the Kerberos v5 protocol for Web applications.

Support for Kerberos authentication is based on other previously defined mechanisms, such as SPNEGO Simple And Protected Negotiate (SPNEGO) [RFC4178] and the Generic Security Services Application Program Interface (GSSAPI).

2. Conventions Used in This Document

The key words "MUST", "MUST NOT", "REQUIRED", "SHALL", "SHALL NOT", "SHOULD", "SHOULD NOT", "RECOMMENDED", "MAY", and "OPTIONAL" are to be interpreted as described in [RFC2119].

3. Access Authentication

\subsection{Reliance on the HTTP/1.1 Specification}

This specification is a companion to the HTTP/1.1 specification [RFC2616], and it builds on the authentication mechanisms defined in [RFC2617]. It uses the augmented BNF section of that document (2.1), and it relies on both the non-terminals defined in that document and other aspects of the HTTP/1.1 specification.

4. HTTP Negotiate Authentication Scheme

Use of Kerberos is wrapped in an HTTP auth-scheme of "Negotiate". The auth-params exchanged use data formats defined for use with the GSS-API [RFC2743]. In particular, they follow the formats set for the SPNEGO [RFC4178] and Kerberos [RFC4121] mechanisms for GSSAPI. The "Negotiate" auth-scheme calls for the use of SPNEGO GSSAPI tokens that the specific mechanism type specifies.

The current implementation of this protocol is limited to the use of SPNEGO with the Kerberos and Microsoft (NT Lan Manager) NTLM protocols.

4.1. The WWW-Authenticate Response Header

If the server receives a request for an access-protected object, and if an acceptable Authorization header has not been sent, the server responds with a "401 Unauthorized" status code, and a "WWWAuthenticate:" header as per the framework described in [RFC2616]. The initial WWW-Authenticate header will not carry any gssapi-data. 
The negotiate scheme will operate as follows:

$$
\begin{array}{ll}
\text { challenge } & =\text { "Negotiate" auth-data } \\
\text { auth-data } & =1 \#(\text { [gssapi-data] ) }
\end{array}
$$

The meanings of the values of the directives used above are as follows:

gssapi-data

If the gss_accept_security_context returns a token for the client, this directive contains the base64 encoding of an initialContextToken, as defined in [RFC2743]. This is not present in the initial response from the server.

A status code 200 status response can also carry a "WWW-Authenticate" response header containing the final leg of an authentication. In this case, the gssapi-data will be present. Before using the contents of the response, the gssapi-data should be processed by gss_init_security_context to determine the state of the security context. If this function indicates success, the response can be used by the application. Otherwise, an appropriate action, based on the authentication status, should be taken.

For example, the authentication could have failed on the final leg if mutual authentication was requested and the server was not able to prove its identity. In this case, the returned results are suspect. It is not always possible to mutually authenticate the server before the HTTP operation. POST methods are in this category.

When the Kerberos Version 5 GSSAPI mechanism [RFC4121] is being used, the HTTP server will be using a principal name of the form of "HTTP/hostname".

\subsection{The Authorization Request Header}

Upon receipt of the response containing a "WWW-Authenticate" header from the server, the client is expected to retry the HTTP request, passing a HTTP "Authorization" header line. This is defined according to the framework described in [RFC2616] and is utilized as follows:

credentials auth-data2

gssapi-data
= "Negotiate" auth-data2

$=1 \#($ gssapi-data ) 
This directive contains the base64 encoding of an InitialContextToken, as defined in [RFC2743].

Any returned code other than a success $2 \mathrm{xx}$ code represents an authentication error. If a 401 containing a "WWW-Authenticate" header with "Negotiate" and gssapi-data is returned from the server, it is a continuation of the authentication request.

A client may initiate a connection to the server with an "Authorization" header containing the initial token for the server. This form will bypass the initial 401 error from the server when the client knows that the server will accept the Negotiate HTTP authentication type.

5. Negotiate Operation Example

The client requests an access-protected document from server via a GET method request. The URI of the document is

"http://www. nowhere.org/dir/index.html".

$$
\text { C: GET dir/index.html }
$$

The first time the client requests the document, no Authorization header is sent, so the server responds with

S: HTTP/1.1 401 Unauthorized

S: WWW-Authenticate: Negotiate

The client will obtain the user credentials using the SPNEGO GSSAPI mechanism type to identify generate a GSSAPI message to be sent to the server with a new request, including the following Authorization header:

$$
\begin{aligned}
& \text { C: GET dir/index.html } \\
& \text { C: Authorization: Negotiate a87421000492aa874209af8bc028 }
\end{aligned}
$$

The server will decode the gssapi-data and pass this to the SPNEGO GSSAPI mechanism in the gss_accept_security_context function. If the context is not complete, the server will respond with a 401 status code with a WWW-Authenticate header containing the gssapi-data.

S: HTTP/1.1 401 Unauthorized

S: WWW-Authenticate: Negotiate 749efa7b23409c20b92356

The client will decode the gssapi-data, pass this into Gss_Init_security_context, and return the new gssapi-data to the server. 
C: GET dir/index.html

C: Authorization: Negotiate 89a8742aa8729a8b028

This cycle can continue until the security context is complete. When the return value from the gss_accept_security_context function

indicates that the security context is complete, it may supply final authentication data to be returned to the client. If the server has more gssapi data to send to the client to complete the context, it is to be carried in a WWW-Authenticate header with the final response containing the HTTP body.

S: HTTP/1.1 200 Success

S: WWW-Authenticate: Negotiate ade0234568a4209af8bc0280289eca

The client will decode the gssapi-data and supply it to gss_init_security_context using the context for this server. If the status is successful from the final gss_init_security_context, the response can be used by the application.

6. Security Considerations

The SPNEGO HTTP authentication facility is only used to provide authentication of a user to a server. It provides no facilities for protecting the HTTP headers or data including the Authorization and WWW-Authenticate headers that are used to implement this mechanism.

Alternate mechanisms such as TLS can be used to provide confidentiality. Hashes of the TLS certificates can be used as channel bindings to secure the channel. In this case clients would need to enforce that the channel binding information is valid. Note that Kerb-TLS [RFC2712] could be used to provide both authentication and confidentiality, but this requires a change to the TLS provider.

This mechanism is not used for HTTP authentication to HTTP proxies.

If an HTTP proxy is used between the client and server, it must take care to not share authenticated connections between different authenticated clients to the same server. If this is not honored, then the server can easily lose track of security context associations. A proxy that correctly honors client to server authentication integrity will supply the "Proxy-support: SessionBased-Authentication" HTTP header to the client in HTTP responses from the proxy. The client MUST NOT utilize the SPNEGO HTTP authentication mechanism through a proxy unless the proxy supplies this header with the "401 Unauthorized" response from the server. 
When using the SPNEGO HTTP authentication facility with clientsupplied data such as PUT and POST, the authentication should be complete between the client and server before sending the user data. The return status from the gss_init_security_context will indicate that the security context is complete. At this point, the data can be sent to the server.

7. Normative References

[RFC2743] Linn, J., "Generic Security Service Application Program Interface Version 2", 2, Update 1", 2743, January 2000.

[RFC2119] Bradner, S., "Key words for use in RFCs to Indicate Requirement Levels", BCP 14, RFC 2119, March 1997.

[RFC4178] Zhu, L., Leach, P., Jaganathan, K., and W. Ingersoll, "The Simple and Protected GSS-API Generic Security Service Application Program Interface (GSS-API) Negotiation Mechanism", 4178, October 2005.

[RFC2616] Fielding, R., Gettys, J., Mogul, J., Frystyk, H., Masinter, L., Leach, P., and T. Berners-Lee, "Hypertext Transfer Protocol -- HTTP/1.1", RFC 2616, June 1999.

[RFC2617] Franks, J., Hallam-Baker, P., Hostetler, J., Lawrence, S., Leach, P., Luotonen, A., and L. Stewart, "HTTP Authentication: Basic and Digest Access Authentication", RFC 2617, June 1999.

[RFC2712] Medvinsky, A. and M. Hur, "Addition of Kerberos Cipher Suites to Transport Layer Security (TLS)", RFC 2712, October 1999 .

[RFC4121] Zhu, L., Jaganathan, K., and S. Hartman, "The Kerberos Version 5 Generic Security Service Application Program Interface (GSS-API) Mechanism: Version 2", RFC 4121, July 2005 . 


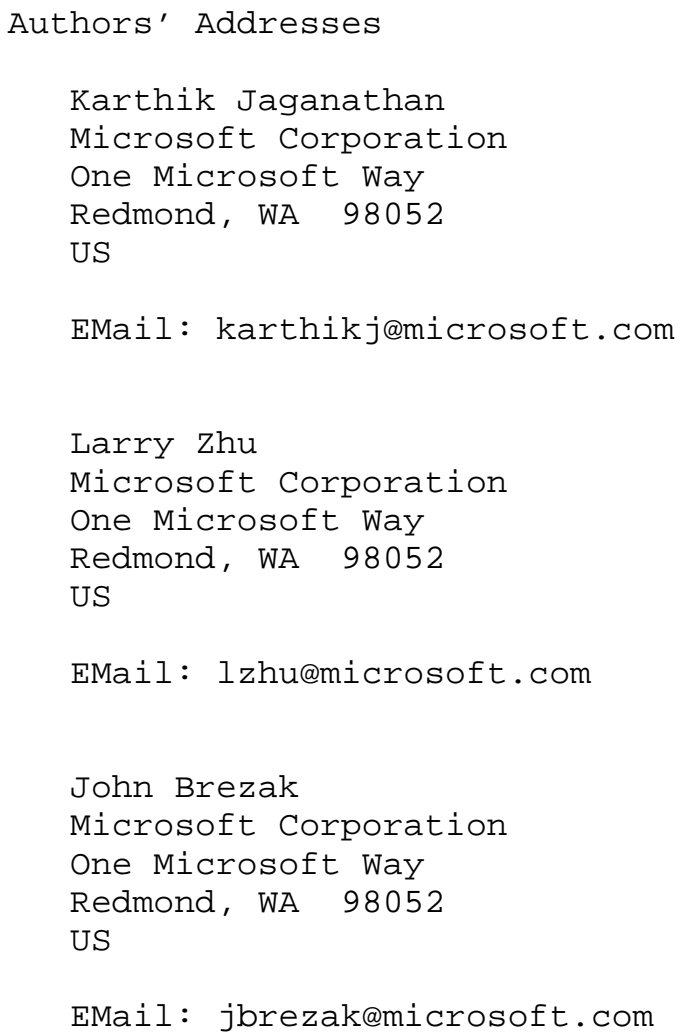


Full Copyright statement

Copyright (C) The Internet Society (2006).

This document is subject to the rights, licenses and restrictions contained in BCP 78 and at www.rfc-editor.org/copyright.html, and except as set forth therein, the authors retain all their rights.

This document and the information contained herein are provided on an "AS IS" basis and THE CONTRIBUTOR, THE ORGANIZATION HE/SHE REPRESENTS OR IS SPONSORED BY (IF ANY), THE INTERNET SOCIETY AND THE INTERNET ENGINEERING TASK FORCE DISCLAIM ALL WARRANTIES, EXPRESS OR IMPLIED, INCLUDING BUT NOT LIMITED TO ANY WARRANTY THAT THE USE OF THE INFORMATION HEREIN WILL NOT INFRINGE ANY RIGHTS OR ANY IMPLIED WARRANTIES OF MERCHANTABILITY OR FITNESS FOR A PARTICULAR PURPOSE.

Intellectual Property

The IETF takes no position regarding the validity or scope of any Intellectual Property Rights or other rights that might be claimed to pertain to the implementation or use of the technology described in this document or the extent to which any license under such rights might or might not be available; nor does it represent that it has made any independent effort to identify any such rights. Information on the procedures with respect to rights in RFC documents can be found in BCP 78 and BCP 79 .

Copies of IPR disclosures made to the IETF Secretariat and any assurances of licenses to be made available, or the result of an attempt made to obtain a general license or permission for the use of such proprietary rights by implementers or users of this specification can be obtained from the IETF on-line IPR repository at http://www.ietf.org/ipr.

The IETF invites any interested party to bring to its attention any copyrights, patents or patent applications, or other proprietary rights that may cover technology that may be required to implement this standard. Please address the information to the IETF at ietf-ipreietf.org.

Acknowledgement

Funding for the RFC Editor function is provided by the IETF Administrative Support Activity (IASA). 